\title{
Evolução da terapêutica de fase aguda em acidente vascular cerebral isquêmico
}

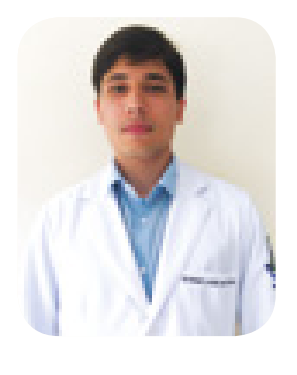

\author{
Daniel Lordelo San Martin¹, Augusto Júnior Azevedo Bastos', \\ Davidson França Pereira', Pedro Antônio Pereira de Jesus ${ }^{1}$
}

\section{RESUMO}

$\mathrm{O}$ acidente vascular cerebral isquêmico (AVCi) define-se como um déficit neurológico súbito com duração maior do que 24 horas. Sua classificação sindrômica tem importantes implicações prognósticas e a classificação etiológica define a conduta em longo prazo. $O$ tratamento da fase aguda vem vivenciando grandes alterações nas últimas três décadas, iniciando com a publicação do primeiro estudo positivo para terapia reperfusional aguda com alteplase venosa em 1995, até três horas do início dos sintomas, e posterior expansão para 4,5 horas em 2008. Outros estudos têm permitido o uso de tenecteplase como terapêutica alternativa se necessário. A evolução da terapêutica inclui os estudos publicados com resultados positivos desde 2015 até 2018 , utilizando a trombectomia mecânica inicialmente em até seis horas e, mais recentemente, permitindo até 24 horas do início dos sintomas, em pacientes selecionados.

PALAVRAS-CHAVE: acidente vascular cerebral isquêmico, trombólise venosa, trombectomia mecânica.

KEY WORDS: ischemic stroke, thrombolysis, mechanical thrombectomy.

\section{INTRODUÇÃO}

$\mathrm{O}$ acidente vascular cerebral isquêmico (AVCi) define-se como um déficit neurológico súbito motivado por isquemia no sistema nervoso central com duração maior do que 24 horas. Atualmente é a maior causa de morte no Brasil e a segunda maior no mundo, com maior prevalência em adultos de meia idade e idosos. Por conta do grande contingente de pacientes que apresentam sequelas importantes e com dependência funcional, o AVCi insere-se num contexto de grande importância clínica. ${ }^{1,2,3}$

A fisiopatologia do AVCi envolve oclusão aguda de artéria que irriga alguma área do sistema nervoso central. Tal área implicada na gênese do evento estará relacionada com os sintomas apresentados pelos pacientes. Há, em termos histológicos, a formação de necrose celular no core arterial e uma área de penumbra isquêmica ao redor do core, que poderá evoluir em maior ou menor grau para necrose na dependência do estabelecimento de reperfusão na área ocluída em função do tempo. Pode haver preservação de função cerebral caso haja o reestabelecimento reperfusional a tempo de salvar o território em risco de isquemia. . $^{3,4}$

Os sintomas de AVCi, de caráter súbito, podem ser associados com a classificação de Oxfordshire, em que há uma divisão anatômica do território arterial agudamente ocluído e o estabelecimento de uma síndrome em função desse território comprometido. A classificação, além de anatômica e funcional, é também prognóstica, dividindo-se em: 1. Circulação Anterior Total: hemiplegia contralateral + novos déficits corticais $(60-80 \%$ mortalidade); 2. Circulação Anterior Parcial: déficit sensorial e motor, associado a déficit cortical ou hemianopsia; 3. Circulação Posterior: déficit motor/sensorial, associado ou não à lesão de nervo craniano ou alteração na acuidade visual; e 4. Síndrome Lacunar: preservação de funções corticais e acometimento sensório-motor puro, e por infartos lacunares até $15 \mathrm{~mm}$ em análise histopatológica. ${ }^{5}$

Há numerosos fatores de risco para AVC. Os principais fatores de risco modificáveis são: hipertensão arterial sistêmica, dislipidemia, diabetes mellitus, sedentarismo e tabagismo. Há ainda os fatores não modificáveis, como: idade $>80$ anos, sexo masculino, história familiar, entre outros. ${ }^{4}$

\section{INVESTIGAÇÃO ETIOLÓGICA}

Ecocardiografia (Transtorácica ou Transesofágica), Doppler de Carótidas e Vertebrais, Angiotomografia Computadorizada de Pescoço e Crânio, Angiorresso- 
nância Magnética de Pescoço e Crânio, ECG de Repouso, Arteriografia Cerebral e Holter são ferramentas de propedêutica complementar utilizadas em maior ou menor grau, cruciais para a definição etiológica do evento isquêmico cerebral e, consequentemente, à sua

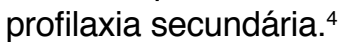

Segundo o TOAST (1993), as causas de AVC isquêmico são: a) aterosclerose de grandes artérias, b) doença de pequenos vasos, c) AVCi cardioembólico, d) outras etiologias por causas determinadas, e) causas indeterminadas. $^{6}$

A aterosclerose de grandes artérias se traduz por uma redução na luz do vaso $\geq 50 \%$, devido à ateromatose do mesmo. A etiologia doença de pequenos vasos caracteriza-se usualmente por síndromes motoras lacunares, intimamente relacionadas ao diabetes mellitus, hipertensão arterial e dislipidemia, porém sem alterações significativas nos exames de investigação que poderiam suscitar outra causa. Já o cardioembólico tem por característica o acometimento de diferentes territórios vasculares, infartos em áreas mais corticais e que tem como determinante uma patologia cardíaca importante - fibrilação atrial, acinesia segmentar, trombo intracavitário, entre outros. Os eventos isquêmicos por outras causas determinadas podem ser vasculites, trombofilias, anemia falciforme. Já a etiologia indeterminada são aqueles AVCis que não foram adequadamente investigados ou apresentam mais de uma etiologia possível, por apresentar alteração em mais de um exame complementar. ${ }^{6} \mathrm{O}$ subtipo criptogênico é uma subdivisão das causas indeterminadas, em que não houve uma associação clara com alteração em exame complementar de investigação. Já foi provada a associação do subtipo criptogênico com fibrilação atrial paroxística e forame oval persistente. ${ }^{7,8}$

\section{ERA PRÉ-REPERFUSIONAL}

Os primeiros trabalhos realizados para a fase aguda do AVCI nos mostram tentativas pouco efetivas de tratar o AVC nesta fase, em alguns casos com a terapêutica instituída, provocando até mesmo dano, no que se refere à morte, recorrência de $\mathrm{AVCl}$ ou independência funcional. Os princípios de fisiopatologia da isquemia cerebral já estavam presentes nesses estudos, faltando, aparentemente, uma adequada seleção de pacientes e terapêutica com maior eficácia.

Um estudo com 19.345 pacientes comparou o uso de aspirina e heparina não fracionada no AVCi agudo. A heparina não fracionada obteve resultados negativos e até mesmo deletérios em doses mais altas e a aspirina obteve resultados positivos quanto à morte $\mathrm{e}$
AVCI recorrente não fatal, porém com resultados pouco expressivos e número necessário para tratar elevado. ${ }^{9}$ Tentou-se ainda, em outro trabalho, averiguar o uso de corticoide no $\mathrm{AVCl}$, pensando-se no racional teórico de diminuir edema cerebral e prevenir aumento da pressão intracraniana, com resultado negativo. ${ }^{10}$

\section{TROMBOLÍTICO VENOSO}

$O$ tratamento da fase aguda tem vivenciado uma evolução muito importante. Não havia nenhuma terapia reperfusional aguda autorizada para o AVC isquêmico até 1995. Foram feitos ensaios clínicos negativos, com altas taxas de hemorragia cerebral, com estreptoquinase e outros. ${ }^{11-13}$ Até que o estudo NINDS atestou o benefício do uso de Alteplase venosa em pacientes com $\mathrm{AVCl}$ até 3 horas de evolução, que não tenham critérios de exclusão absolutos (tabela 1). Os resultados (figura 1) mostraram uma maior taxa de independência funcional (tabela 2 - Rankin Scale Modificada) no grupo intervenção. ${ }^{14}$ Essa variação de tempo do ictus do déficit neurológico agudo permaneceu até 2008, quando foi publicado o estudo ECASS III, estendendo o período até 4 horas e 30 minutos. ${ }^{15}$

Novos estudos foram feitos e outros estudos estão em andamento para permitir o uso de um novo trombolítico venoso: o tenecteplase, que inclusive possui posologia mais rápida e fácil. O estudo NOR-TEST mostrou que os resultados são semelhantes no que se refere à eficácia e segurança, comparando-se à Alteplase, porém o estudo foi negativo, pois foi uma análise de superioridade. ${ }^{16}$ Já o estudo EXTEND-IA TNK, de 2018, atesta que o tenecteplase foi associado com maior incidência de reperfusão e melhores desfechos funcionais do que Alteplase (figura 2) em pacientes com $\mathrm{AVCl}$ encaminhados para trombectomia mecânica após trombólise (respeitando-se o limite de 4,5h e os critérios de inclusão já estabelecidos para trombectomia). ${ }^{17}$

\section{TERAPIA TROMBOLÍTICA INTRA-ARTERIAL}

Conjecturou-se a hipótese de realizar trombolíticos intra-arteriais para $\mathrm{AVCl}$, porém, com resultados questionáveis. A prourokinase intra-arterial (PROACT) foi testada em um estudo de 180 pacientes com AVCI há menos de 6 horas, com oclusão de artéria cerebral média $(A C M)$, com Rankin Scale $\leq 2$. Obteve resultados positivos quanto à funcionalidade, porém, observou-se uma alta taxa de sangramento intracraniano sintomático $(10 \%)$ no grupo intervenção..$^{18}$ Outro estudo conhecido, o MELT de 2007, obteve resultados negativos no seu desfecho primário e maior mortalidade e hemorragia intracraniana sintomática. ${ }^{19}$ 
Tendo em vista esses estudos relatados e outros estudos desanimadores, o uso rotineiro de trombolíticos intra-arteriais para AVCI foi desencorajado. Começou-se então a buscar outras soluções, entrando na era dos estudos de trombectomia mecânica no AVCI.

\section{TROMBECTOMIA MECÂNICA INTRA-ARTERIAL}

Os primeiros estudos utilizando essas técnicas foram negativos. Ambos de 2013, o SYNTHESIS (362 pacientes) e o IMS III (656 pacientes) obtiveram resultados desanimadores. O SYNTHESIS comparou terapia intra-arterial sem trombólise venosa, sendo o grupo-controle terapia padrão - trombólise venosa, em pacientes com AVCl até $4,5 \mathrm{~h}$ do ictus, enquanto no IMS III foram incluídos na intervenção pacientes com AVCI até $3 h$ do ictus e que tinham sido submetidos à trombólise venosa. A terapia intra-arterial era feita com trombolíticos intra-arteriais, incluindo alteplase ou trombectomia mecânica com devices de primeira geração. Postula-se que os resultados foram negativos justamente por ter incluído os trombolíticos intra-arteriais e esses devices mais antigos, além de menor experiência de neurorradiologista e a não utilização de exames de imagem para incluir apenas pacientes com oclusões proximais de grandes vasos. ${ }^{20,21}$

O MR CLEAN (2015) foi o primeiro estudo que utilizou trombectomia mecânica com resultados positivos. Ensaio clínico randomizado, cego para desfechos, envolveu 502 pacientes com AVCl até 6 horas do ictus com oclusão de artéria carótida interna $(\mathrm{ACl})$ intracraniana distal, $A C M$, segmentos $M 1$ ou $M 2$, artéria cerebral anterior (ACA), segmentos $A 1$ ou $A 2$. Utilizou, em sua grande maioria ( $82 \%)$, o stent retrievable de segunda geração após trombólise venosa, comparado com terapia trombolítica venosa padrão, e foram vistos resultados de desfecho primário positivos, estatisticamente significantes, quanto à funcionalidade em 90 dias e com boas taxas de segurança. ${ }^{22}$ Novos estudos foram lançados seguindo a mesma linha de pesquisa e resultados ${ }^{23-26}$ e uma metanálise agrupou-os estatisticamente. Os critérios gerais dessa metanálise foram: diagnóstico clínico de AVC agudo $\leq 12$ horas (porém, a grande maioria dos estudos só permitia até 6 horas) e idade $\geq 18$ anos, com um infarto pequeno e área de penumbra isquêmica passível de preservação, oclusão arterial intracraniana da $\mathrm{ACl}$ distal, ou segmentos M1 ou M2 da ACM, ou segmentos A1 ou A2 da ACA, vistas em AngioTC, angioRM ou angiografia por subtração digital. Os resultados apresentaram maior independência funcional, com boa segurança, inclusive para aqueles que utilizaram ou não a alteplase venosa antes da trombectomia (figura 3 ). ${ }^{27}$

Muitos pacientes se apresentam à emergência fora dessa janela terapêutica de 6 horas, principalmente os denominados wake-up stroke, pacientes que estavam dormindo e acordaram com o déficit, não podendo precisar ao certo o horário que o mesmo ocorreu. A necessidade de novos estudos era imperiosa. O estudo DAWN (2018) envolveu 206 pacientes com AVCi de 6 até 24 horas do ictus, tendo oclusão da $\mathrm{ACl}$ intracraniana ou $\mathrm{ACM}$ M1, vista em Angio TC/RM, além de $\leq 1 / 3$ infartado do território da ACM, pacientes com NIH-SS $\geq 10$ e área de penumbra isquêmica a ser salva. Esse estudo utilizou os stents retrievable de segunda geração em $98 \%$ do grupo intervenção. Os resultados foram positivos para o grupo intervenção (figura 4): independência funcional em 90 dias: $49 \%$ x 13\% (diferença ajustada 33 IC 95\% 21-44). Desfechos secundários relativos à segurança, semelhantes em ambos os grupos. ${ }^{28}$ Já o estudo DEFUSE 3 realizou a trombectomia naqueles pacientes com ictus de 6 até 16 horas, com critérios muito semelhantes ao DAWN, e também mostrou resultados positivos em relação à terapia médica padrão até então. ${ }^{29}$

\section{CONCLUSÃO}

A análise pormenorizada e baseada em evidências do estado da arte atual permite-nos afirmar que a trombólise venosa com alteplase e tenecteplase pode ser feita em até 4,5 horas do início do $\mathrm{AVCl}$, respeitando-se os critérios definidos nos estudos já citados. A terapia trombolítica intra-arterial não obteve resultados animadores e deve ser desencorajada. Por sua vez, a trombectomia mecânica é uma realidade mundial, já atestada por diversos estudos, tendo sido recentemente ampliado o intervalo de tempo de 6 para 24 horas para os pacientes que se incluam nos critérios estabelecidos.

\section{FIGURAS E TABELAS}

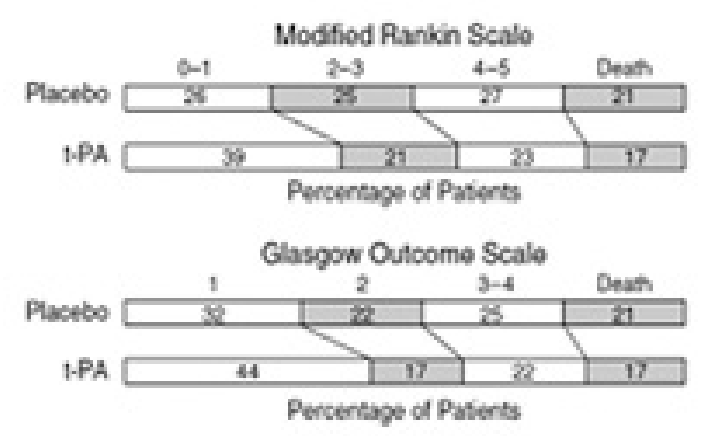

Figura 1 - Desfecho em termos da escala Rankin Modificada e de desfecho de Glasgow em pacientes tratados com alteplase até 3 horas. (Adaptado de: Brott T, et al. Tissue Plasminogen Activator for Acute Ischemic Stroke. N Engl J Med 1995;333:1581). 
Table 2. Outcomes.

\begin{tabular}{|c|c|c|c|c|}
\hline Outcome & $\begin{array}{l}\text { Tenecteplase Group } \\
\qquad(\mathbb{N}=101)\end{array}$ & $\begin{array}{l}\text { Alteplase Group } \\
\qquad(\mathrm{N}=101]\end{array}$ & Ellect Sine $(95 \% \mathrm{CI})$ & P Value \\
\hline \multicolumn{5}{|l|}{ Primary efficacy eutcome } \\
\hline $\begin{array}{l}\text { Substantial reperfusion at initial angiegraphic assessment } \\
\qquad-n 0 .(\%)^{*}\end{array}$ & $22(22)$ & $20(10)$ & & \\
\hline Difference - percentage points & & & $12(2-21)$ & 0.002 \\
\hline Adjusted incidense ratio & & & $2.2(1.2-4.4)$ & 0.03 \\
\hline Adjussed odds ratio & & & $2.6(1.2-5.9)$ & 0.02 \\
\hline \multicolumn{5}{|l|}{ Secondary outcomes } \\
\hline \multicolumn{5}{|l|}{ Score on the modified Rarkin scale at 90 days $t$} \\
\hline Median seore (IQR) on ordinal analysist? & $2(0-3)$ & $3(1-4)$ & $1.7(1.0-2.8)$ & 0.04 \\
\hline Functionally independent cutcome - no. (As)S & $65(6-4)$ & $52(51)$ & & \\
\hline Adjusted incidence ratio & & & $1.2(1.0-1.5)$ & 0.06 \\
\hline Adjusted odds ratio & & & $1.8(1.0-3.4)$ & 0.05 \\
\hline Exceilent outcome - na. $[\%] S$ & $\$ 2(\$ 1)$ & $43(43)$ & & \\
\hline Adjusted incidence ratio & & & $1.2(0.9-1.6)$ & 0.20 \\
\hline Adjusted odds ratio & & & $1.4(0.8-2.6)$ & 0.23 \\
\hline
\end{tabular}

Figura 2 - Desfecho quanto à reperfusão e escala de Rankin Modificada no estudo EXTEND-IA TNK, testando tenecteplase $\mathrm{x}$ alteplase em AVCl. (Adaptado de Campbell BCV, et al. Tenecteplase versus Alteplase before Thrombectomy for Ischemic Stroke. N Engl J Med 2018; 378:1573-1582).

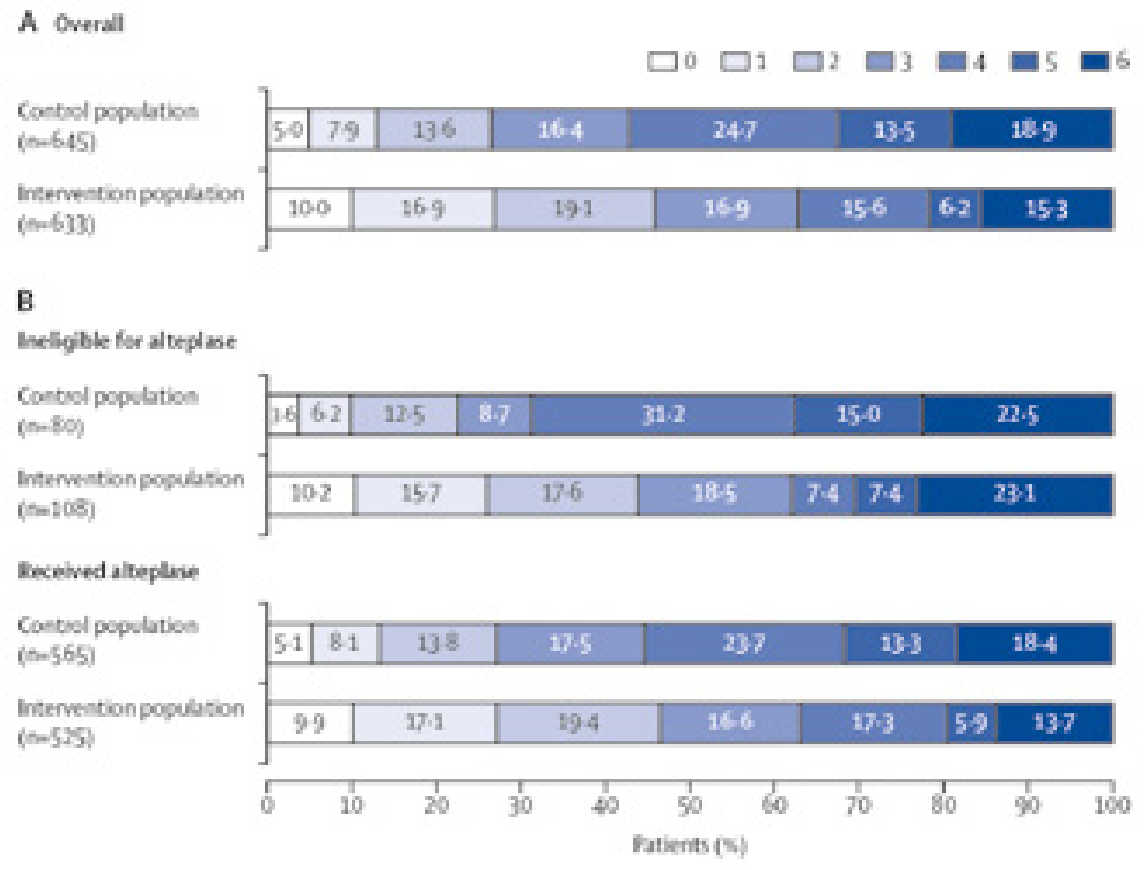

Figura 3 - Escala de Rankin Modificada na metanálise envolvendo cinco artigos acerca da trombectomia mecânica no AVCI. (Adaptado de Goyal M, et al. Endovascular thrombectomy after large-vessel ischaemic stroke: a meta-analysis of individual patient data from five randomised trials. Lancet 2016;387(10029):1723-31). 

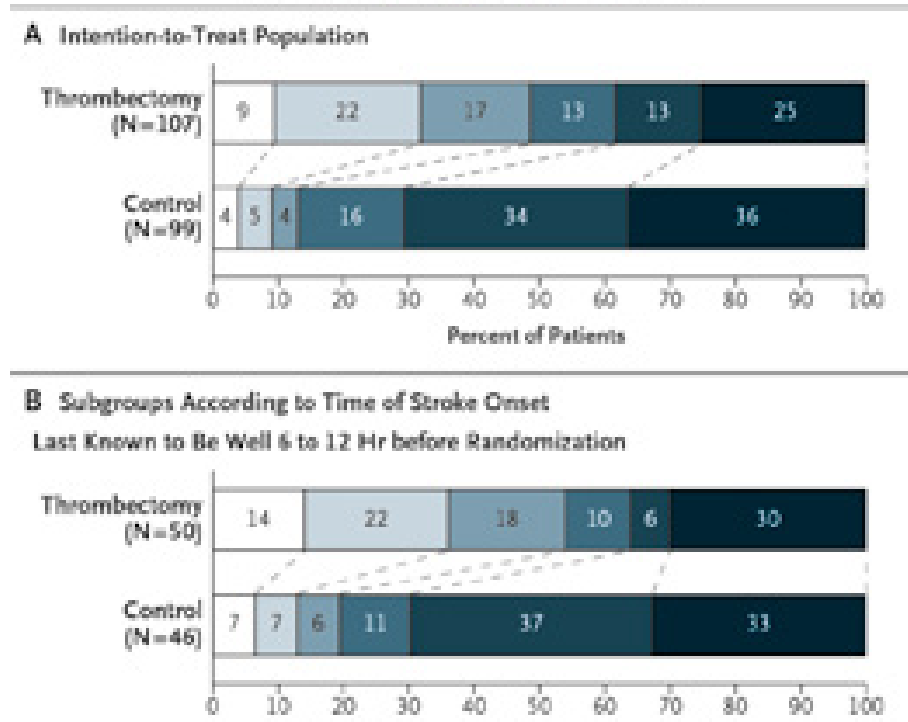

Last Known to Be Well $>12$ to $24 \mathrm{Hr}$ befoee Randomization

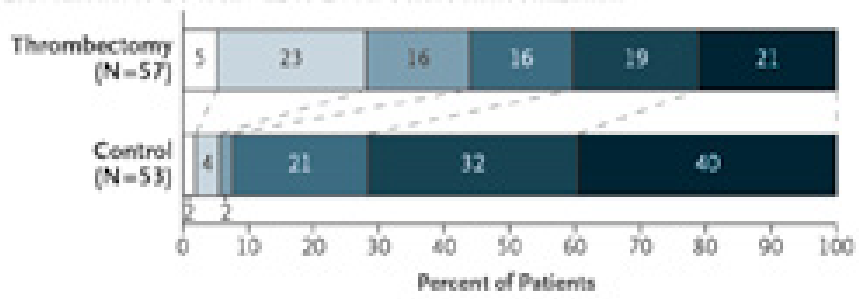

Figura 4 - Escala de Rankin Modificada nos pacientes que realizaram trombectomia mecânica no AVCl com sintomas iniciados de 6 até 24 horas. (Adaptado de Nogueira RG, et al. Thrombectomy 6 to 24 Hours after Stroke with a Mismatch between Deficit and Infarct. N Engl J Med 2018; 378:11-21.)

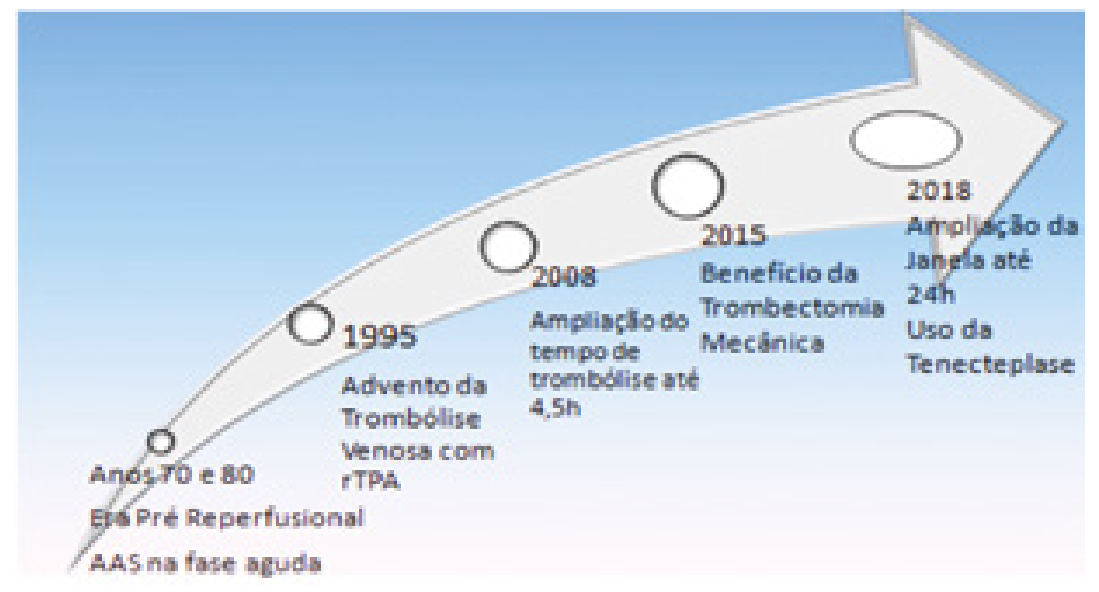

Figura 5 - Evolução Temporal da Terapêutica Aguda em AVCi 
Tabela 1 - Critérios de exclusão para trombólise venosa em Acidente Vascular Cerebral Isquêmico.

\begin{tabular}{l}
\hline Critérios de Inclusão \\
\hline Clínica de AVC, TC sem achados ou compatíveis com AVCi, causando déficit neurológico mensurável \\
\hline \hline Idade $\geq 18$ anos \\
\hline Sintomas iniciados ou paciente visto bem pela última vez há $<3 \mathrm{~h}$ \\
\hline AVC isquêmico ou trauma cranioencefálico grave nos três meses anteriores \\
\hline Hemorragia intracraniana prévia \\
\hline Neoplasia intracraniana intra-axial \\
\hline Malignidade gastrointestinal ou hemorragia nos 21 dias anteriores \\
\hline Cirurgia intracraniana ou intra-espinhal nos três meses anteriores \\
\hline Sintomas sugestivos de hemorragia subaracnóide \\
\hline Elevação persistente da pressão arterial (PAS $\geq 185 \mathrm{mmHg}$ ou PAD $\geq 110 \mathrm{mmHg})$ \\
\hline Sangramento interno ativo, suspeita de dissecção de aorta ou endocardite infecciosa \\
\hline Discrasia sanguínea/Contagem de plaquetas $<100.000 / \mathrm{mm} 3 / \mathrm{NR}>1,7$ ou TP> 15s ou TTPa $>40 \mathrm{~s} /$ \\
Doses terapêuticas de heparina de baixo peso molecular \\
\hline Uso atual de inibidor direto da trombina ou inibidor direto do fator Xa \\
\hline TC de crânio com evidência de hemorragia ou hipodensidade extensa \\
\hline \hline
\end{tabular}

Tabela 2 - Modified Rankin Scale

\begin{tabular}{cl}
\hline \hline Escala & \multicolumn{1}{c}{ Quadro clínico } \\
\hline 0 & Ausência de sintomas. \\
\hline 1 & $\begin{array}{l}\text { Apesar dos sintomas, ausência de comprometimento funcional significante; capaz de } \\
\text { executar atividades e funções usuais. }\end{array}$ \\
\hline \hline 2 & $\begin{array}{l}\text { Comprometimento leve da funcionalidade; incapaz de executar todas as atividades } \\
\text { prévias, mas capaz de realizar os cuidados pessoais sem assistência. }\end{array}$ \\
\hline \hline 3 & $\begin{array}{l}\text { Comprometimento moderado da funcionalidade; precisa de ajuda, contudo, é capaz de } \\
\text { andar sozinho. }\end{array}$ \\
\hline \hline 5 & $\begin{array}{l}\text { Comprometimento moderadamente severo; incapaz de andar e do autocuidado sem } \\
\text { assistência. }\end{array}$ \\
\hline \hline 6 & $\begin{array}{l}\text { Funcionalidade severamente comprometida; acamado; incontinência; necessitando de } \\
\text { cuidado e atenção constantes. }\end{array}$ \\
\hline \hline
\end{tabular}




\section{REFERÊNCIAS}

1. World Health Organization. (WHO). The top 10 causes of death. [Internet] Geneva; 2014. [Cited in 2018 May]. Available from: http://www.who.int/mediacentre/factsheets/fs310/em.

2. Brasil. Ministério da saúde. Informações de saúde TABNET - Estatísticas vitais. Datasus. [internet]. [citado em Maio 2018] Disponível em: http://tabnet. datasus.gov.br

3. Deb P, Sharma S, Hassan KM. Pathophysiologic mechanisms of acute ischemic stroke: An overview with emphasis on therapeutic significance beyond thrombolysis. Pathophysiology 2010; 17:197-218.

4. Oliveira-Filho J, Martins SCO, Pontes-Neto OM, et al. Guidelines for acute ischemic stroke treatment Part I. Arq Neuropsiquiatr 2012;70(8):621-629.

5. Bamford J, Sandercock P, Dennis M, et al. Classification and natural history of clinically identifiable subtypes of cerebral infarction. Lancet 1991; 337 : 1521-26.

6. Adams Jr. HP, Bendixen BH, Kappelle LJ, et al. Classification of Subtype of Acute Ischemic Stroke Definitions for Use in a Multicenter Clinical Trial. Stroke 1993;24(1):35.

7. Sanna T, Diener HC, Passman RS, et al. Cryptogenic Stroke and Underlying Atrial Fibrillation. N Engl J Med 2014; 370:2478-2486.

8. Handke, M; Harloff A; Olschewski M, et al. Patent Foramen Ovale and Cryptogenic Stroke in Older Patients. N Engl J Med 2007; 357:2262-2268

9. Sandercock P, Collins R, Counsell C. The International Stroke Trial (IST): a randomised trial of aspirin, subcutaneous heparin, both, or neither among 19.435 patients with acute ischaemic stroke. Lancet 1997; 349: 1569-81.

10. Bauer RB, Tellez H. Dexamethasone as Treatment in Cerebrovascular Disease. 2. A Controlled Study in Acute Cerebral Infarction. Stroke 1973; 4:547-55.

11. Fletcher AP, Alkjaersig N, Lewis $\mathrm{M}$, et al. A Pilot Study of Urokinase Therapy in Cerebral Infarction. Stroke 1976; 7(2):135-142.

12. Donnan GA, Davis SM, Chambers BR, et al. Streptokinase for acute ischemic stroke with relationship to time of administration. JAMA 1996;276:961-966.

13. Multicenter Acute Stroke Trial European Study Group. Thrombolytic therapy with streptokinase in acute ischemic stroke. N Engl J Med 1996;335:145150.

14. Brott $\mathrm{T}$, Broderick $\mathrm{J}$, Kothari $\mathrm{R}$, et al. Tissue Plasminogen Activator for Acute Ischemic Stroke. N Engl J Med 1995;333:1581.
15. Hacke W, Kaste M, Bluhmki E, et al. Thrombolysis with Alteplase 3 to 4.5 Hours after Acute Ischemic Stroke. N Engl J Med 2008;359:1317-1329.

16. Logallo N, Novotny V, Assmus J, et al. Tenecteplase versus alteplase for management of acute ischaemic stroke (NOR-TEST): a phase 3, randomised, open-label, blinded endpoint trial. Lancet Neurol. 2017; 16(10):781-788

17. Campbell BCV, Mitchell PJ, Churilov L, et al. Tenecteplase versus Alteplase before Thrombectomy for Ischemic Stroke. N Engl J Med 2018; 378:15731582

18. Furlan A, Higashida R, Wechsler $L$, et al. The PROACT II Study: A Randomized Controlled Trial. JAMA. 1999;282(21):2003-2011.

19. Ogawa A, Mori E, Minematsu K, et al. Randomized trial of intraarterial infusion of urokinase within 6 hours of middle cerebral artery stroke: the middle cerebral artery embolism local fibrinolytic intervention trial (MELT) Japan. Stroke. 2007 Oct;38(10):2633-9.

20. Ciccone A, Valvassori $L$, Nichelatti $M$, et al. Endovascular Treatment for Acute Ischemic Stroke. N Engl J Med 2013; 368:904-913.

21. Broderick JP, Palesch YY, Demchuk AM, et al. Endovascular Therapy after Intravenous t-PA versus t-PA Alone for Stroke. N Engl J Med 2013; 368:893903

22. Berkhemer OA, Fransen PS, Beumer D, et al. A randomized trial of intraarterial treatment for acute ischemic stroke. N Engl J Med. 2015;372(1):11.

23. Goyal M, Demchuk AM, Menon BK, et al. Randomized assessment of rapid endovascular treatment of ischemic stroke. N Engl J Med. 2015;372(11):1019.

24. Saver JL, Goyal M, Bonafe A, et al. Stent-retriever thrombectomy after intravenous t-PA vs. t-PA alone in stroke. N Engl J Med. 2015;372(24):2285.

25. Campbell BC, Mitchell PJ, Kleinig TJ, et al. Endovascular therapy for ischemic stroke with perfusion-imaging selection. N Engl J Med. 2015;372(11):1009.

26. Jovin TG, Chamorro A, Cobo E, et al. Thrombectomy within 8 hours after symptom onset in ischemic stroke. N Engl J Med. 2015;372(24):2296.

27. Goyal M, Menon BK, van Zwam WH, et al. Endovascular thrombectomy after large-vessel ischaemic stroke: a meta-analysis of individual patient data from five randomised trials. Lancet 2016;387(10029):172331

28. Nogueira RG, Jadhav AP, Haussen DC. Thrombectomy 6 to 24 Hours after Stroke with a Mismatch between Deficit and Infarct. N Engl J Med 2018; 378:11-21. 
29. Albers, GW, Marks MP, Kemp S. Thrombectomy for Stroke at 6 to 16 Hours with Selection by Perfusion Imaging. N Engl J Med 2018; 378:708-718.

1- Serviço de Neurologia do HSI.

Endereço para correspondência:

daniel_lordelo@hotmail.com 